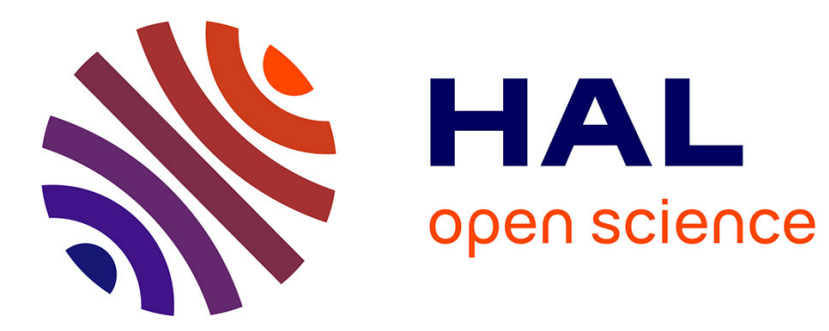

\title{
Optical emissions associated with terrestrial gamma ray flashes
}

\author{
Wei Xu, Sébastien Celestin, Victor P. Pasko
}

\section{To cite this version:}

Wei $\mathrm{Xu}$, Sébastien Celestin, Victor P. Pasko. Optical emissions associated with terrestrial gamma ray flashes. Journal of Geophysical Research Space Physics, 2015, 120, pp.1355-1370. 10.1002/2014JA020425 . insu-01182137

\section{HAL Id: insu-01182137 https://hal-insu.archives-ouvertes.fr/insu-01182137}

Submitted on 30 Jul 2015

HAL is a multi-disciplinary open access archive for the deposit and dissemination of scientific research documents, whether they are published or not. The documents may come from teaching and research institutions in France or abroad, or from public or private research centers.
L'archive ouverte pluridisciplinaire HAL, est destinée au dépôt et à la diffusion de documents scientifiques de niveau recherche, publiés ou non, émanant des établissements d'enseignement et de recherche français ou étrangers, des laboratoires publics ou privés. 


\section{Journal of Geophysical Research: Space Physics}

\author{
RESEARCH ARTICLE \\ 10.1002/2014JA020425 \\ Key Points: \\ - Results of fluorescence emissions \\ from air agree with laboratory \\ observations \\ - Distribution of electrons in the \\ full energy range for two TGF \\ mechanisms \\ - Model-based quantifications of \\ optical emissions generated during \\ TGF production
}

\section{Correspondence to:}

W. Xu,

wxx5015@psu.edu

\section{Citation:}

Xu, W., S. Celestin, and V. P. Pasko

(2015), Optical emissions asso-

ciated with terrestrial gamma

ray flashes, J. Geophys. Res.

Space Physics, 120, 1355-1370,

doi:10.1002/2014JA020425.

Received 23 JUL 2014

Accepted 31 DEC 2014

Accepted article online 8 JAN 2015

Published online 4 FEB 2015

\section{Optical emissions associated with terrestrial gamma ray flashes}

\author{
Wei Xu' ${ }^{1}$, Sebastien Celestin ${ }^{2}$, and Victor P. Pasko' \\ ${ }^{1}$ Communications and Space Sciences Laboratory, Department of Electrical Engineering, Pennsylvania State University, \\ University Park, Pennsylvania, USA, ${ }^{2}$ Laboratory of Physics and Chemistry of the Environment and Space, University of \\ Orleans, CNRS, Orleans, France
}

Abstract Terrestrial gamma ray flashes (TGFs) are high-energy photon bursts produced by high-energy electrons originating in the Earth's atmosphere through bremsstrahlung processes. In this paper, we present modeling studies on optical emissions resulting from the excitation of air molecules produced by the large population of electrons involved in TGF events based on two possible production mechanisms: relativistic runaway electron avalanches (RREAs) and acceleration of thermal runaway electrons produced by high-potential intracloud lightning leaders. Numerical models developed in this study are first validated through the calculation of fluorescence emissions from air excited by energetic electrons and comparison with available laboratory observations. Detailed discussion of the role of excitation and ionization collisions on the formation of the electron energy distribution is presented. Moreover, using Monte Carlo simulations, we show that electron energy distributions established from the two TGF production mechanisms considered here are inherently different over the full energy range. The strong energy dependence of the capability of electrons to generate excited states responsible for optical emissions from neutral and ionized nitrogen molecules leads to intrinsic differences in optical emissions produced by different mechanisms of TGF production. We also show that TGFs are most likely accompanied by detectable levels of optical emissions and that the distinct optical features are of significant interest for constraining and validating current TGF production models.

\section{Introduction}

Brief and intense photon bursts that originate from the Earth's atmosphere, known as terrestrial gamma ray flashes (TGFs), were first discovered in 1994 by Fishman et al. [1994] using the Burst and Transient Source Experiment detector aboard the Compton Gamma-Ray Observatory. After their discovery, TGFs have been detected by the Reuven Ramaty High Energy Solar Spectroscopic Imager (RHESSI) [Smith et al., 2005], the Fermi Gamma ray Space Telescope [Briggs et al., 2010], and the Astrorivelatore Gamma a Immagini Leggero satellite [Marisaldi et al., 2010]. Spaceborne measurements indicate that TGFs typically consist of single or multiple pulses, last from a few tens of microseconds to a few milliseconds [e.g., Fishman et al., 2011], and exhibit energy spectra that may extend up to $100 \mathrm{MeV}$ [e.g., Tavani et al., 2011].

In addition to satellite observations, radio signals radiated by TGF-associated lightning flashes have been used to infer the conditions that are required for the production of this high-energy phenomenon. Observational evidence shows that TGFs are closely associated with the initial development stages of normal polarity intracloud lightning that transports negative charge upward (+IC) [e.g., Stanley et al., 2006; Shao et al., 2010; Lu et al., 2010]. Moreover, Marshall et al. [2013] have performed dedicated analyses on the initial breakdown (IB) stage of $10 \mathrm{IC}$ flashes that emitted radio signals similar to TGF-producing ones and speculated that avalanches of relativistic runaway electrons caused IB pulses.

Two main mechanisms have been proposed and developed for interpreting TGF observations. The first mechanism is that of relativistic runaway electron avalanches (RREAs), which involves acceleration and multiplication of relativistic seed electrons (e.g., cosmic ray secondary electrons) under the application of large spatial scale electric fields within thunderstorms [Gurevich et al., 1992; Dwyer and Smith, 2005]. Since natural background radiation or extensive air showers have been shown to provide insufficient number of seed electrons for the RREA theory to explain the TGF fluences observed from satellites, relativistic feedback mechanisms of positrons and X-rays have been invoked [Dwyer, 2008]. The other proposed mechanism is based on production of thermal runaway electrons [Gurevich, 1961] by the negative corona flash stages of stepping lightning leaders [Moss et al., 2006; Celestin and Pasko, 2011]. Modeling studies by Xu et al. 


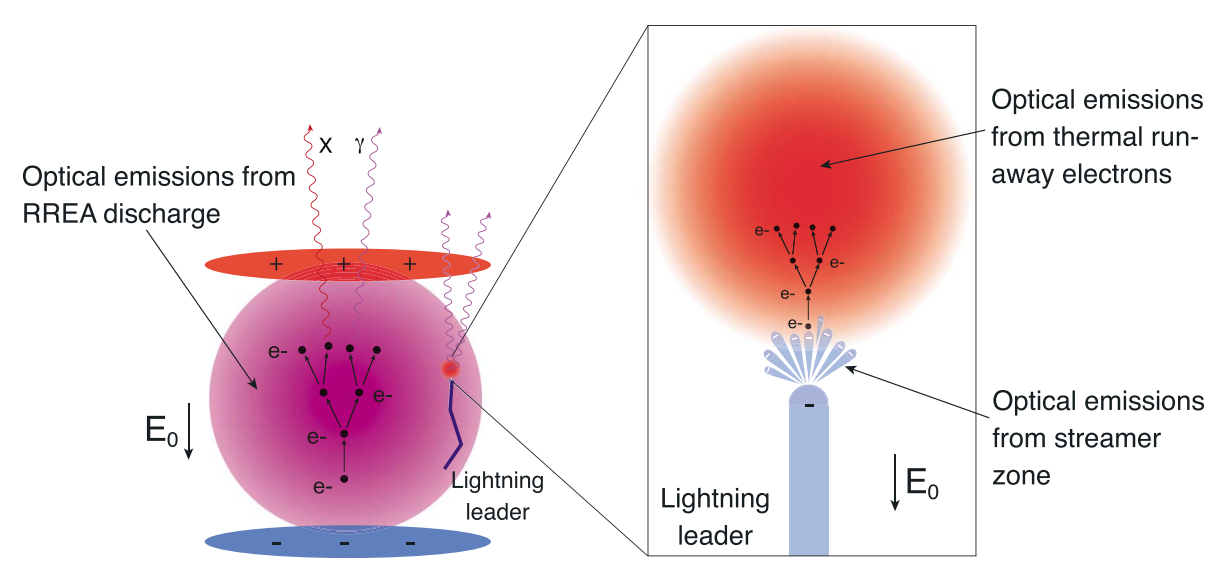

RREA in thunderstorm, large-scale homogeneous electric field

Thermal runaway electrons in the leader field

Figure 1. Illustration of optical emissions produced by two TGF production mechanisms: RREAs in the large-scale homogeneous electric field within thunderstorms and the acceleration of thermal runaway electrons under the highly inhomogeneous electric field produced around the tip region of $+\mathrm{IC}$ lightning leaders during the negative corona flash stage.

[2012] and Celestin et al. [2012] support this mechanism by demonstrating that further acceleration of these thermal runaway electrons in the electric field produced near the tip region of long unbranched +IC lightning leaders leads to results consistent with satellite measurements of TGF spectra. The two production mechanisms, including the associated optical emissions and the fan-shaped streamer zone, in front of the lightning leader, are illustrated in Figure 1.

Although photon energy spectra established from both mechanisms show good agreement with satellite measurements [e.g., Dwyer and Smith, 2005; Xu et al., 2012; Celestin et al., 2012], there are notable differences between these two mechanisms. Lightning flashes do not play a critical role in the RREA theory and the high-energy parts of the electron distribution and corresponding photon spectrum are weakly dependent on the magnitude of the homogeneous electric field driving the RREAs [e.g., Babich et al., 2004; Dwyer and Smith, 2005], whereas the energy spectrum of gamma rays emitted during the acceleration of thermal runaway electrons in the highly inhomogeneous electric fields around lightning leader tips is influenced by the physical properties and spatial electric field configurations associated with lightning stepped leaders [Xu et al., 2012; Celestin et al., 2012].

In spite of numerous experimental and theoretical efforts, little observational evidence is available about the TGF source. One of the most important unknowns is whether and which measurable optical emissions are generated during the production of TGFs. Optical emissions usually provide insightful knowledge about the energetics of electrons and the driving electric field in atmospheric discharges [e.g., Celestin and Pasko, 2010a] and are, therefore, extensively used in the studies of thunderstorm-related Transient Luminous Events (TLEs) at high altitudes in the Earth's atmosphere [e.g., Kuo et al., 2005, 2009; Liu et al., 2006; Adachi et al., 2006]. Recently, Østgaard et al. [2013] have reported optical emissions, detected for the first time, by the Lightning Imaging Sensor on the Tropical Rainfall Measuring Mission, from TGF-associated IC flashes. It is also worth mentioning that, based on high-speed camera observations, it has been suggested in Stolzenburg et al. [2013] that the impulsive breakdown associated with initial leaders during IB stages of cloud-to-ground (CG) and IC flashes can generate considerable amount of visible light. Furthermore, Dwyer et al. [2013] quantified optical emissions produced by relativistic feedback discharges based on the RREA mechanism. These studies motivate the present work that aims at the development of an experimentally testable framework that would allow to distinguish between different production mechanisms of TGF events by quantifying the optical emissions generated by the large population of high- and low-energy electrons.

To achieve this goal, we first introduce a full energy range relativistic Monte Carlo model to study the dynamics of electrons and the characteristic electron energy distributions in both TGF production mechanisms (section 2.1) and another numerical model for quantifying the associated optical emissions 
(sections 2.2 and 2.3). In section 3.1, we validate our models by comparing results of fluorescence emissions excited by energetic electrons with available laboratory observations. Then, we present simulation results of electron energy distributions in the full energy range obtained in the cases of homogeneous electric fields (section 3.2.1) and a strongly inhomogeneous electric field produced by high-potential lightning leaders (section 3.2.2). We quantify optical emissions that can be possibly generated during production of TGFs in section 3.3. We further analyze in section 4.1 the concept of fluorescence efficiency in the context of TGFs and discuss the scaling of optical emissions with air density/altitude in the Earth's atmosphere in section 4.2. Finally, we discuss the implications of these calculations for understanding the mechanisms behind TGFs in section 4.3.

\section{Model Formulation}

\subsection{Monte Carlo Model for Electrons}

The Monte Carlo model used in the present study to simulate the propagation and collisions of electrons in air is similar to that described in Celestin and Pasko [2011]. This model is three-dimensional (3-D) in the velocity space, 3-D in the configuration space, relativistic, and simulates electrons from sub-eV to GeV. The elastic scattering of high-energy $(>500 \mathrm{eV})$ electrons by nitrogen and oxygen molecules is modeled using a shielded-Coulomb cross section, similar to that described in Dwyer [2007]. The singly differential cross sections of $\mathrm{N}_{2}$ and $\mathrm{O}_{2}$ are calculated over the full range of energy using the relativistic binaryencounter-bethe (RBEB) model [Kim et al., 2000; Celestin and Pasko, 2010b]. In particular, this model enables orbital description of the differential cross section of ionization collisions for all target electrons. The energies of secondary electrons after ionization collisions are obtained through the knowledge of this differential cross section, and the scattering angles of primary and secondary electrons are determined from the relativistic equations of conservation of momentum and energy considering that the newly formed ion is static.

The driving electric field is a critical parameter in the present study as it directly controls the avalanche processes of runaway electrons. In the case of TGF production by thermal runaway electrons, the method of moments [Balanis, 1989, p. 670] is first used in order to calculate the electric field in the vicinity of a $+\mathrm{IC}$ lightning negative leader tip during the corona flash associated with the stepping of the leader (see Celestin and Pasko [2011], and references therein for discussion of the related phenomenology). The electric potential of the lightning leader tip with respect to the ambient potential can be approximated by $U_{1}=E_{0} I / 2$ [Bazelyan and Raizer, 2000, p. 54], where $E_{0}$ is magnitude of ambient electric fields and $l$ is the length of an unbranched leader channel. A representative electric potential that provides consistent results with RHESSI cumulative energy spectra has been suggested to be greater than $100 \mathrm{MV}$ [Xu et al., 2012]. For such high potential, the ambient large-scale thunderstorm electric field is assumed to be $E_{0}=5 \times 10^{4} \mathrm{~V} / \mathrm{m}$ [e.g., Marshall et al., 2001], the radius of the leader channel is chosen as $1 \mathrm{~cm}$ [Rakov and Uman, 2003, section 4.4.6, p. 134], and the IC lightning length is $I=4 \mathrm{~km}$. Note that it has recently been shown in Pasko [2014] that, using electrostatic modeling, such a long unbranched leader can also explain magnitudes of the dipole moment changes and current moment changes believed to be associated with TGFs.

We study the dynamics of electrons in the full energy range in either large-scale homogeneous electric field producing RREAs or highly inhomogeneous electric field produced around the lightning leader tip region during negative corona flash stages. Most importantly, by keeping track of the acceleration of runaway electrons and the rapid generation of low-energy secondary electrons in both mechanisms, we focus on providing a rigorous description of the electron energy distribution in the full energy range.

Monte Carlo simulation encounters difficulty when modeled electrons cover many decades in the energy space, for instance, from sub-eV to GeV, for mainly two reasons. First, the copious amount of low-energy secondary electrons generated via ionization collisions of high-energy electrons severely increases the complexity of the simulation. Second, the simulation time that is required for the ensemble of electrons to achieve a statistical steady state is dramatically prolonged because of the high collision rate of low-energy electrons. In order to overcome these difficulties, the calculation of electron energy distribution is divided into two parts: a low-energy part (from sub-eV to $10 \mathrm{keV}$ ) and a high-energy part (from $10 \mathrm{keV}$ to GeV). After steady state is reached in both parts, distribution function of electrons over the full range of energy is then obtained by normalizing the high-energy part calculation in order to connect to the low-energy part at $10 \mathrm{keV}$. This technique relies on adopting two characteristic time steps to maintain the collisional frequencies of high-energy and low-energy electrons separately. It is also supported by the similarity of 
the low-energy parts of energy distributions for electron swarms initiated by either low-energy electrons or high-energy runaway electrons [Colman et al., 2010]. This technique not only allows precise descriptions for both high- and low-energy electrons, but also greatly shortens the computation time. We have carefully validated this technique by comparing the results with full energy range simulations for representative test cases.

\subsection{Optical Emissions Model}

The main energy loss for runaway electrons occurs through ionization collisions with air molecules, resulting in low-energy secondary electrons. These primary and secondary electrons are able to generate excited species via impact excitation, and optical emissions can be generated by the radiative relaxation of these excited species. The model employed for evaluating optical emissions is similar to that documented by Liu and Pasko [2004]. Modeling optical emissions from the first positive band systems of $\mathrm{N}_{2}\left(B^{3} \Pi_{g} \rightarrow A^{3} \Sigma_{u}^{+}, 1 \mathrm{PN}_{2}\right)$ is complex, particularly for cases at high air pressures with continuous excitation by electrons. The populating of $\mathrm{N}_{2}\left(B^{3} \Pi_{g}\right)$ involves processes of direct impact excitation by electrons, cascading from higher electronic states of $\mathrm{N}_{2}$, intersystem collisional transfer with adjacent states [e.g., Morrill and Benesch, 1996], and energy-pooling reactions [e.g., Piper, 1988]. Besides, the deexcitation of $\mathrm{N}_{2}\left(B^{3} \Pi_{g}\right)$ by $\mathrm{N}_{2}$ molecules is strongly vibrational-level-dependent [e.g., Piper, 1988; Morrill and Benesch, 1996]. Given that the focus of the present study is the fluorescence light produced by TGF sources at low altitudes where $\mathrm{N}_{2}\left(B^{3} \Pi_{g}\right)$ is heavily quenched and $1 \mathrm{PN}_{2}$ emissions are weak, in the present work, we mainly focus on optical emissions originating from the second positive band system of $\mathrm{N}_{2}\left(C^{3} \Pi_{u} \rightarrow B^{3} \Pi_{g^{\prime}} 2 \mathrm{PN}_{2}\right)$ and the first negative band system of $\mathrm{N}_{2}^{+}\left(B^{2} \Sigma_{u}^{+} \rightarrow X^{2} \Sigma_{g}^{+}, 1 \mathrm{NN}_{2}^{+}\right)$.

The intensity of optical emissions in Rayleighs is given by the following expression [Chamberlain, 1978, p. 213]:

$$
I_{k}=10^{-6} \int_{L^{\prime}} A_{k} n_{k} \mathrm{~d} l
$$

where $n_{k}\left[1 / \mathrm{cm}^{3}\right]$ is the number density of excited species in state $k, A_{k}$ is the radiation transition rate and the typical values for optical emissions from $2 \mathrm{PN}_{2}$ and $1 \mathrm{NN}_{2}^{+}$are, respectively, $2 \times 10^{7} \mathrm{~s}^{-1}$ and $1.4 \times 10^{7} \mathrm{~s}^{-1}$ [Vallance Jones, 1974, p. 119]. The integral is taken along $L^{\prime}(\mathrm{cm})$, representing the horizontal line of sight.

The quantity $n_{k}$ is governed by processes including direct excitation by electrons, cascading from higher-energy electronic states, and collisional quenching by air molecules. The number density $n_{k}$ can be calculated using the following relation [Sipler and Biondi, 1972]:

$$
\frac{\partial n_{k}}{\partial t}=-\frac{n_{k}}{\tau_{k}}+\sum_{m} n_{m} A_{m}+v_{k} n_{e}
$$

where $\tau_{k}=\left[A_{k}+\alpha_{1} N_{\mathrm{N}_{2}}+\alpha_{2} N_{\mathrm{O}_{2}}\right]^{-1}$ is the total lifetime of state $k_{1} \alpha_{1}$ and $\alpha_{2}$ are the quenching rate coefficients due to collisions with $\mathrm{N}_{2}$ and $\mathrm{O}_{2}$ molecules, respectively, $N_{\mathrm{N}_{2}}$ and $\mathrm{N}_{\mathrm{O}_{2}}$ are, respectively, the number densities of $\mathrm{N}_{2}$ and $\mathrm{O}_{2}$ molecules, the sum over the terms $n_{m} A_{m}$ represents cascading from higher-energy states, $v_{k}$ is the excitation frequency for generating the upper excited states responsible for emissions from $2 \mathrm{PN}_{2}$ and $1 \mathrm{NN}_{2}^{+}$, and $n_{e}$ is the number density of electrons. For the sake of simplicity, steady state assumption is applied in order to find out $n_{k}$. This is valid given that the lifetimes of the upper excited states responsible for $2 \mathrm{PN}_{2}$ and $1 \mathrm{NN}_{2}^{+}$emissions are very short (less than several microseconds [Vallance Jones, 1974, p. 119]) when compared to the characteristic time scale of the TGF sources considered in this paper.

The excitation frequency, describing contributions to $n_{k}$ from direct electronic excitation by electrons, is consistently calculated using Monte Carlo simulation results. The quantity $v_{k}$, defined as the number of molecular species $j$ in the excited state $k$ produced in unit time per one electron, is given by

$$
v_{k}=N_{j} \int \sigma_{k}(\varepsilon) v(\varepsilon) f(\varepsilon) \mathrm{d} \varepsilon
$$

where $N_{j}$ is the number density of unexcited species $j, \sigma_{k}(\varepsilon)$ is the cross section for the excitation of state $k$, which depends on the kinetic energy of the incident electron $\varepsilon, v(\varepsilon)$ is the velocity of the colliding electron with an energy $\varepsilon$ defined as $v(\varepsilon)=c \sqrt{1-\left[1+\varepsilon /\left(m_{e} c^{2}\right)\right]^{-2}}$, where $c$ is the speed of light and $m_{e}$ is the electron rest mass, and $f(\varepsilon)$ is the electron energy distribution function, which is normalized so that $\int_{0}^{+\infty} f(\varepsilon) \mathrm{d} \varepsilon=1$. Electron impact excitation cross sections used for determining the excitation frequency 
of generating the upper excited states of $2 \mathrm{PN}_{2}$ are taken from BOLSIG+ database [Hagelaar and Pitchford, 2005]. These cross sections are provided between the threshold energy and $1 \mathrm{keV}$. However, for the energy distributions considered here, their values for energies $>100 \mathrm{eV}$ are small and their effects are negligible (see Figure $5 b)$. Concerning $1 \mathrm{NN}_{2}^{+}$, it is considered that an $\mathrm{N}_{2}^{+}\left(B^{2} \Sigma_{u}^{+}\right)$ion is produced if one of the two $\left(2 \sigma_{u}\right)$ electrons from the nitrogen molecule is removed [e.g., Van Zyl and Pendleton, 1995]. Thus, an accurate representation for the ionization cross section of $\left(2 \sigma_{u}\right)$ electrons from the threshold energy to hundred $\mathrm{MeV}$ is needed. The RBEB model, due to its orbital-based description for the differential ionization cross section, is therefore well suited for the calculation of the excitation frequency of $\mathrm{N}_{2}^{+}\left(B^{2} \Sigma_{u}^{+}\right)$leading to $1 \mathrm{NN}_{2}^{+}$emissions. Indeed, the branching ratio fraction for producing $\mathrm{N}_{2}^{+}\left(B^{2} \Sigma_{u}^{+}\right)$during ionization reactions of $\mathrm{N}_{2}$ molecules by $100 \mathrm{eV}$ electrons calculated using the RBEB model yields 0.163 and agrees well with the value $0.145 \pm 0.019$ suggested by Van Zyl and Pendleton [1995].

Collisional quenching processes are critical at the source altitudes of TGFs [e.g., Dwyer and Smith, 2005; Xu et al., 2012], which are far below the quenching altitudes of both $2 \mathrm{PN}_{2}$ and $1 \mathrm{NN}_{2}^{+}$[e.g., Liu et al., 2006]. In the present study, we consider that $\mathrm{N}_{2}\left(C^{3} \Pi_{u}\right)$ can be quenched by collisions with $\mathrm{N}_{2}$ and $\mathrm{O}_{2}$ molecules with rate coefficients of $10^{-11} \mathrm{~cm}^{3} / \mathrm{s}$ [Kossyi et al., 1992] and $3 \times 10^{-10} \mathrm{~cm}^{3} / \mathrm{s}$ [Vallance Jones, 1974, p. 119], respectively. The primary quenchers of $\mathrm{N}_{2}^{+}\left(B^{2} \Sigma_{u}^{+}\right)$are $\mathrm{N}_{2}$, with a rate coefficient of $4.53 \times 10^{-10} \mathrm{~cm}^{3} / \mathrm{s}$ [e.g., Mitchell, 1970; Kuo et al., 2005; Pancheshnyi et al., 1998], and $O_{2}$, with a rate coefficient of $7.36 \times 10^{-10} \mathrm{~cm}^{3} / \mathrm{s}$ [e.g., Mitchell, 1970; Kuo et al., 2005; Pancheshnyi et al., 1998]. It is important to note that optical emissions evaluated using the modeling approach adopted in this paper significantly rely on the choice of quenching scheme and corresponding rate coefficients.

As in Dwyer et al. [2013], aurora spectra of $2 \mathrm{PN}_{2}$ and $1 \mathrm{NN}_{2}^{+}$presented in Vallance Jones [1974] are used as an approximate spectral distribution for fluorescence photons to investigate the wavelength dependence of optical features. We note that our present calculations do not take into account the effects of radiative transfer between the source of emission and the observer.

\subsection{Characteristic Radial Dimensions}

The characteristics of optical emissions that can be possibly generated during TGF production, including intensities and intensity ratios between optical band systems, mostly depend on the energy distribution and number density of electrons. In this work, except for the comparison with laboratory observations in section 3.1, we assume a uniform spherically symmetric spatial distribution for source electrons involved in TGFs. The number density of electrons is then obtained using the full energy distribution and an assumption that $10^{17}$ electrons with energy above $1 \mathrm{MeV}$ are generated during TGFs, that is believed to be required for explaining TGF fluences and spectra [Dwyer and Smith, 2005]. Although this assumption is not accurate enough for capturing the morphological characteristics of TGF-associated optical emissions, it does not have a significant impact on quantifying the total intensities of optical emissions. The characteristic radial dimensions representing processes of RREAs, acceleration and multiplication of thermal runaway electrons in the electric field produced by the $100 \mathrm{MV}$ lightning leader, and the streamer zone of the $100 \mathrm{MV}$ lightning leader are discussed below, schematically depicted in Figure 1 and summarized in Table 2.

In RREAs, propagation over multiple runaway avalanche lengths is required for relativistic electrons to gain energies on the order of tens of MeV and to explain TGF spectra, corresponding to an acceleration distance of approximately $1 \mathrm{~km}$ for typical electric fields in thunderclouds [e.g., Dwyer et al., 2012]. In contrast, the electric field produced by a $100 \mathrm{MV}$ lightning leader is highly inhomogeneous, intense close to the leader tip, and weak far from it [e.g., Celestin et al., 2012]. This electric field is able to accelerate thermal runaway electrons over relatively short distances and a representative acceleration distance is found to be approximately $50 \mathrm{~m}$ for a $100 \mathrm{MV}$ lightning leader.

Additionally, in this work, the intensity of optical emissions radiated from the streamer zone of the $100 \mathrm{MV}$ lightning leader is also estimated using the streamer modeling results presented in Liu et al. [2008] and the knowledge about the number of streamers and the size of the streamer zone. Specifically, the number of streamers present in a streamer zone is $N_{s}=Q_{s} / q_{s}$ [e.g., Bazelyan and Raizer, 2000; Celestin and Pasko, 2011], where $Q_{s}$ is the total electric charge contained in the streamer zone, and $q_{s}$ is the average charge carried by a streamer, typically on the order of $1 \mathrm{nC}$ [Bazelyan and Raizer, 2000, p. 69-71]. As pointed out by Celestin and Pasko [2011], $Q_{s}$ is quadratically dependent on the electric potential difference formed by 


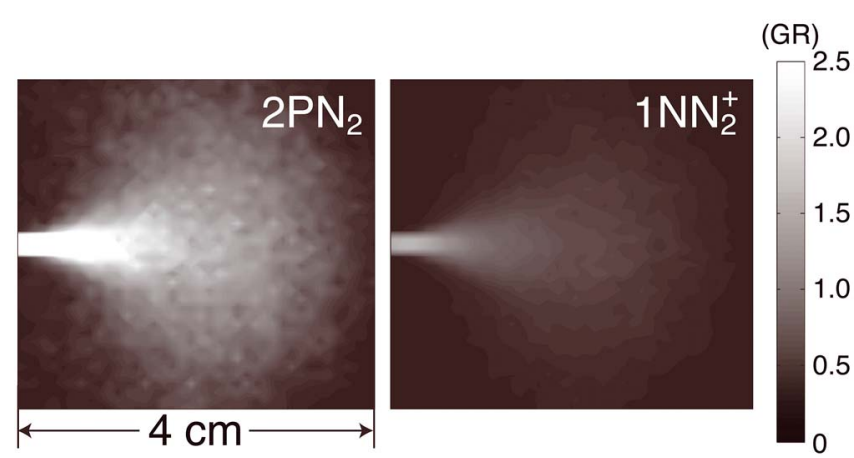

Figure 2. Simulation results of fluorescence radiation from air at ground pressure $\left(N_{0} \simeq 2.688 \times 10^{25} \mathrm{~m}^{-3}\right)$ excited by a continuous beam of $50 \mathrm{keV}$ electrons. Fluorescence emissions exhibit a conical shape. The diameter of this beam, best represented by the strongest emissions from $2 \mathrm{PN}_{2}$, is approximately $4 \mathrm{~cm}$. lightning leaders with respect to ambient potential $\left(U_{\mid}\right)$and can be calculated as follows:

$$
Q_{\mathrm{s}}=\frac{\pi \varepsilon_{0} U_{1}^{2}}{2 E_{\mathrm{s}}^{-}}
$$

where $E_{\mathrm{s}}^{-}$is the electric field in the steamer zone of negative leaders and its value is taken as $12.5 \mathrm{kV} / \mathrm{cm}$ [Babaeva and Naidis, 1997, Figure 7]. From these relations, we estimate that, for long unbranched lightning leaders with electric potential of $100 \mathrm{MV}$, the total number of streamers constituting the streamer zone should be on the order of $10^{8}$. As for the characteristic size of the streamer zone, it is proportional to $U_{1}: R_{\mathrm{sz}}^{-}=U_{1} / 2 E_{\mathrm{s}}^{-}$. Therefore, the radius of the streamer zone in front of a $100 \mathrm{MV}$ lightning leader is approximately $40 \mathrm{~m}$ at ground level.

\section{Results}

\subsection{Comparison With Laboratory Observations}

In order to perform a validation of the present numerical models, fluorescence emissions produced by energetic electrons are first calculated in the framework of Monte Carlo simulation and compared to laboratory observations of Davidson and O'Neil [1964] and Nagano et al. [2004]. One of the advantages that is afforded by the current Monte Carlo model is that it is capable of accurately describing and recording the spatial and temporal information for all the collisions taking place in the system. Owing to this advantage, an analog of photograph of fluorescence from air generated by a continuous beam of $50 \mathrm{keV}$ electrons at ground pressure can be derived from first principles and is presented in Figure 2. As clearly shown in this figure, these fluorescence emissions exhibit a conical shape, following the spatial distribution of electrons, and the diameter of this beam, best represented by the strongest emissions from $2 \mathrm{PN}_{2}$, is approximately

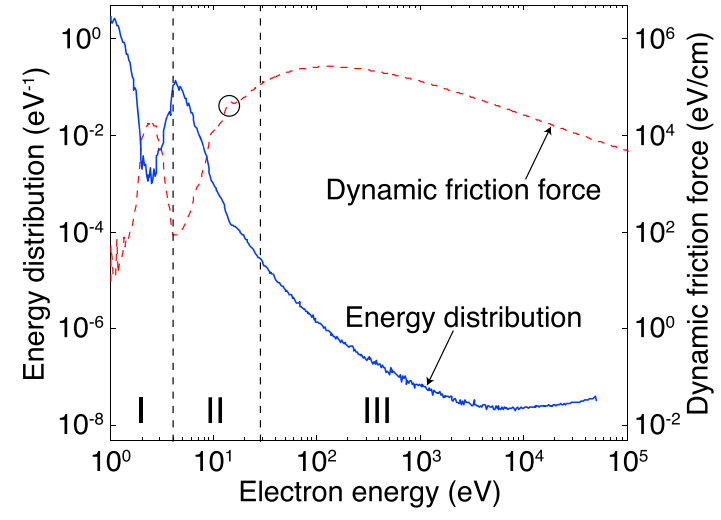

Figure 3. Steady state energy distribution of a continuous beam of $50 \mathrm{keV}$ electrons in air at 600 torr. Also shown is the dynamic friction force of electrons at ground pressure due to collisions with air molecules computed using the set of cross sections adopted in Monte Carlo simulation (red dashed line). The dominant energy loss processes in regions I, II, and III, are vibrational and rotational excitations, electronic excitation, and ionization collisions, respectively. The circle around $14.1 \mathrm{eV}$ represents the enhancement in the dynamic friction force, mainly arising from energy losses to the electronic excitation of $\mathrm{N}_{2}\left(C^{3} \Pi_{u}\right)$, that is also reflected in the electron energy distribution.
$4 \mathrm{~cm}$. The morphological features of these optical emissions, including the conical shape and the size of the illuminated region, are in excellent agreement with experimental observations [Davidson and O'Neil, 1964].

In the absence of external electric fields, the energy distribution, especially in the low-energy range (up to a few tens of eV), is characterized by the dynamic friction force of electrons. This point can be readily illustrated by a direct comparison of the dynamic friction force of electrons resulting from collisions with air molecules at ground pressure and the steady state electron energy distribution produced by the continuous beam of $50 \mathrm{keV}$ electrons, as shown in Figure 3. This energy distribution is calculated by adopting a convolution technique. In particular, we first model the temporal evolution of the deceleration process undergone by an ensemble of $50 \mathrm{keV}$ electrons due to collisions with air molecules in the case that they are injected instantaneously into air at 600 torr. 

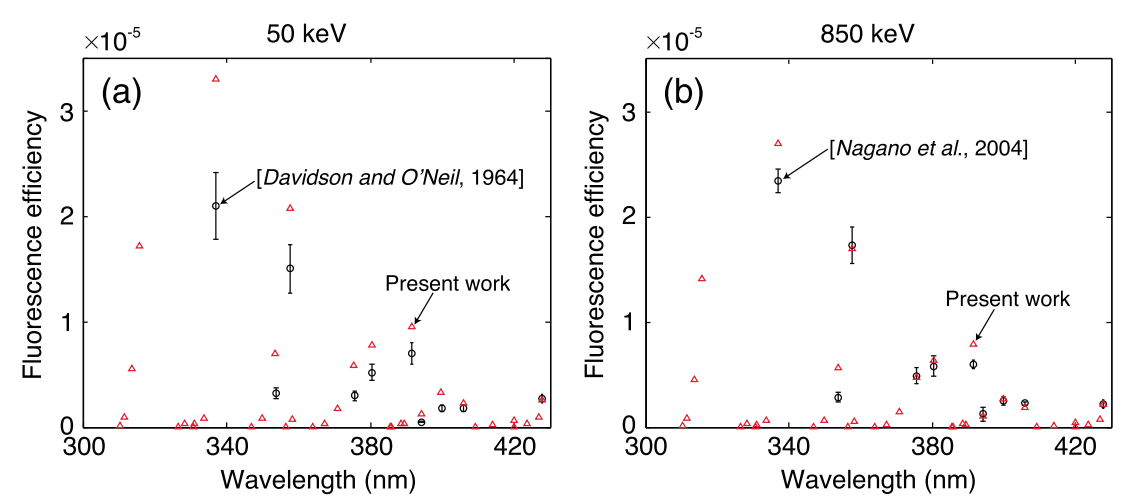

Figure 4. Comparison of fluorescence efficiency from air at 600 torr for major emission bands of $2 \mathrm{PN}_{2}$ and $1 \mathrm{NN}_{2}^{+}$, in the wavelength range between $300 \mathrm{~nm}$ and $430 \mathrm{~nm}$, excited by (a) $50 \mathrm{keV}$ electrons between experimental measurements of Davidson and O'Neil [1964] and present modeling results; (b) $850 \mathrm{keV}$ electrons between experimental measurements of Nagano et al. [2004] and present modeling results.

Namely, we obtain the impulse response of the system. The steady state energy distribution of the continuous $50 \mathrm{keV}$ electron beam is then calculated by convolving over the temporal evolution of this electron pulse. We have verified that directly modeling a continuous beam of $50 \mathrm{keV}$ electrons in the simulation leads to identical results, while being a lot more time consuming. From Figure 3 , we see that the depletion in the electron energy distribution around $2.5 \mathrm{eV}$ precisely pinpoints the first peak of the dynamic friction force, defined by strong vibrational excitations of nitrogen molecules, while the peak of electron energy distribution around $4 \mathrm{eV}$ coincides with a trough in the dynamic friction force. Furthermore, enhancement around $14.1 \mathrm{eV}$ in the dynamic friction force (marked by a circle in Figure 3), that mainly comes from energy losses to the electronic excitation of $\mathrm{N}_{2}\left(C^{3} \Pi_{u}\right)$, is reflected in the energy distribution.

In the region I marked in Figure 3, where the electron energy is below $\sim 4 \mathrm{eV}$, vibrational and rotational excitations are the dominant energy loss processes. In particular, vibrational excitations facilitate fast removal of electrons from this energy region and accordingly cause the precipitous decrease in the energy distribution from $1 \mathrm{eV}$ to $\sim 2.5 \mathrm{eV}$. Region II is delineated by electrons with energy between $\sim 4 \mathrm{eV}$ and $\sim 28 \mathrm{eV}$. Electronic excitation is the principal channel of energy loss in this region and the cross sections of electronic excitation collisions for generating the upper excited states of $2 \mathrm{PN}_{2}$ peak in this region. In region $\mathrm{III}$, electrons possess energy in excess of the binding energy of electrons in $\mathrm{N}_{2}$ and $\mathrm{O}_{2}$ molecules and mostly convert their kinetic energy into the generation of secondary electrons, namely, ionization collisions.

Fluorescence efficiency is defined in the studies of extensive air showers [Bunner, 1967] as the fraction of the total energy deposited during the passage of electrons in a gas that is eventually transferred into fluorescence photons. For the comparison with Nagano et al. [2004], fluorescence efficiency is calculated by dynamically recording the number of fluorescence photons generated and the amount of energy deposited by $850 \mathrm{keV}$ electrons in Monte Carlo simulations. However, in contrast with the thin target technique used in Nagano et al. [2004], Davidson and O'Neil [1964] employed a thick target technique to measure the fluorescence efficiency of $50 \mathrm{keV}$ electrons by injecting a continuous electron beam into the gas chamber. In order to compare with the experimental measurements in Davidson and O'Neil [1964], we first calculate the production of fluorescence photons in the wavelength range between 300 and $430 \mathrm{~nm}$ and the energy deposition into air molecules by an ensemble of $50 \mathrm{keV}$ electrons in the case that they are injected instantaneously into air at 600 torr. Fluorescence emissions corresponding to the steady state of continuous injection are then obtained by convolving the fluorescence pulse produced by the instantaneous injection of $50 \mathrm{keV}$ electrons and assuming the same injection current as that in Davidson and O'Neil [1964]. Fluorescence efficiency is further determined as the ratio of the energy released in fluorescence photons to the energy deposited by the incident electron beam.

The comparison with wavelength-resolved measurements [Davidson and O'Neil, 1964; Nagano et al., 2004 ] is shown in Figure 4 and documented in Table 1. Our modeling results are generally in very good agreement with laboratory measurements of Nagano et al. [2004] for both $2 \mathrm{PN}_{2}$ and $1 \mathrm{NN}_{2}^{+}$band systems. 
Table 1. Comparison of Fluorescence Efficiency $\left(\times 10^{-5}\right)$ From Air at 600 Torr for Major Emission Bands of $2 \mathrm{PN}_{2}$ and $1 \mathrm{NN}_{2}^{+}$, in the Wavelength Range Between $300 \mathrm{~nm}$ and $430 \mathrm{~nm}$, Excited by Energetic Electrons Between Experimental Measurements of Davidson and O'Neil [1964] ${ }^{\mathrm{a}}$ (50 keV electrons), Nagano et al. [2004] (850 keV electrons), and Present Modeling Results

\begin{tabular}{lcccc} 
Wavelength (A) & $\begin{array}{c}\text { Davidson and O'Neil [1964] } \\
\text { Nagano et al. [2004] }\end{array}$ & & \\
\hline $3371,2 \mathrm{PN}_{2}(0-0)$ & $50 \mathrm{keV}$ & $850 \mathrm{keV}$ & $50 \mathrm{keV}$ & $850 \mathrm{keV}$ \\
$3537,2 \mathrm{PN}_{2}(1-2)$ & 2.10 & $2.23-2.45$ & 3.306 & 2.703 \\
$3577,2 \mathrm{PN}_{2}(0-1)$ & 0.32 & $0.237-0.331$ & 0.695 & 0.568 \\
$3756,2 \mathrm{PN}_{2}(1-3)$ & 1.50 & $1.56-1.90$ & 2.076 & 1.697 \\
$3805,2 \mathrm{PN}_{2}(0-2)$ & 0.30 & $0.415-0.563$ & 0.582 & 0.476 \\
$3914,1 \mathrm{NN}_{2}^{+}(0-0)$ & 0.52 & $0.480-0.680$ & 0.783 & 0.640 \\
$3943,2 \mathrm{PN}_{2}(2-5)$ & 0.70 & $0.558-0.638$ & 0.951 & 0.791 \\
$3998,2 \mathrm{PN}_{2}(1-4)$ & 0.05 & $0.058-0.188$ & 0.131 & 0.107 \\
$4059,2 \mathrm{PN}_{2}(0-3)$ & 0.18 & $0.212-0.286$ & 0.333 & 0.272 \\
$4278,1 \mathrm{NN}_{2}^{+}(0-1)$ & 0.18 & $0.209-0.243$ & 0.230 & 0.188 \\
\hline
\end{tabular}

${ }^{\text {a}}$ The systematic error for the measurements of Davidson and O'Neil [1964] is $\pm 15 \%$.

This agreement also validates our use of aurora spectra in order to quantify the wavelength dependence of optical emissions produced by energetic electrons. In addition, we note that the mean energy loss of $850 \mathrm{keV}$ electrons derived from Monte Carlo simulation is approximately $1.74 \mathrm{keV} / \mathrm{cm}$, close to the theoretical value defined by the dynamic friction force at this pressure. The slight difference observed for the (0-0) transition of $1 \mathrm{NN}_{2}^{+}$between calculated and experimental results, at $391 \mathrm{~nm}$, is likely due to three-body deactivation processes as pointed out in Mitchell [1970] and Nagano et al. [2004], which are able to weaken the efficiency by approximately $20 \%$. As for the fluorescence efficiency produced by continuous injection of $50 \mathrm{keV}$ electrons, our modeling results also agree with experimental measurements of Davidson and $\mathrm{O}^{\prime} \mathrm{Neil}$ [1964] for $1 \mathrm{NN}_{2}^{+}$, while the agreement is not as good for $2 \mathrm{PN}_{2}$ and we note that, for some bands of $2 \mathrm{PN}_{2}$ (i.e., $2 \mathrm{PN}_{2}(1-2)$ ), as large as a factor of 2 differences are observed.

\subsection{Distribution of Electrons in the Full Energy Range}

3.2.1. Relativistic Runaway Electron Avalanches

Figure 5a shows the distribution of electrons in the full energy range produced by the acceleration and multiplication of relativistic runaway electrons in a large-scale homogeneous reduced electric field of $E / N=70 \mathrm{Td}\left(\sim 18.8 \mathrm{kV} / \mathrm{cm}\right.$ at $\left.N \simeq 2.688 \times 10^{25} \mathrm{~m}^{-3}, 1 \mathrm{Td}=10^{-21} \mathrm{Vm}^{2}\right)$ in air at ground pressure. This energy
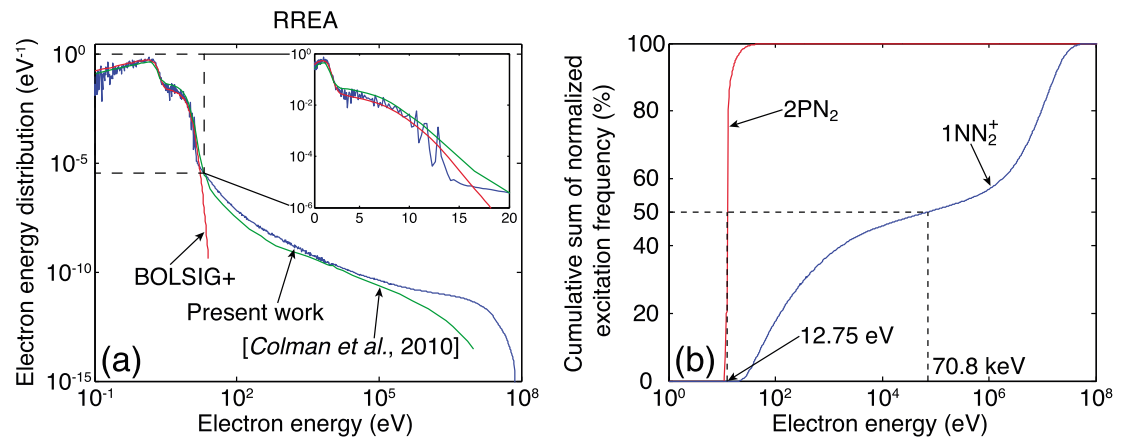

Figure 5. (a) Distribution of electrons in the full energy range produced by the acceleration and multiplication of relativistic runaway electrons in a large-scale homogeneous electric field of $70 \mathrm{Td}(18.8 \mathrm{kV} / \mathrm{cm})$. The results are obtained in air at ground level $\left(N_{0} \simeq 2.688 \times 10^{25} \mathrm{~m}^{-3}\right)$. Also presented are BOLSIG+ calculations [Hagelaar and Pitchford, 2005] and modeling results presented in Colman et al. [2010, Figure 11]. The inset is a zoom-in view of the energy range below $20 \mathrm{eV}$. (b) Cumulative sum of the frequency for generating $\mathrm{N}_{2}\left(C^{3} \Pi_{u}\right)$ and $\mathrm{N}_{2}^{+}\left(B^{2} \Sigma_{u}^{+}\right)$excited by the large population of electrons in RREA processes. Fifty percent of the upper excited states of $\mathrm{N}_{2}$ responsible for $2 \mathrm{PN}_{2}$ emissions is produced by electrons with energy below $12.75 \mathrm{eV}$, while $50 \%$ of the upper excited states of $\mathrm{N}_{2}^{+}$responsible for $1 \mathrm{NN}_{2}^{+}$emissions is produced by electrons with energy below $70.8 \mathrm{keV}$. 

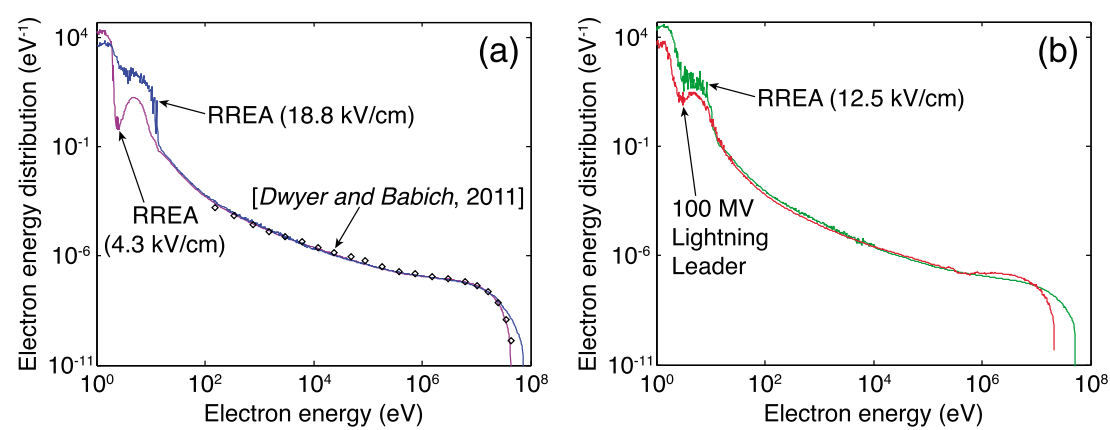

Figure 6. Distribution of electrons in the full energy range produced by the acceleration and multiplication of (a) relativistic runaway electrons in homogeneous electric fields: $4.3 \mathrm{kV} / \mathrm{cm}$ and $18.8 \mathrm{kV} / \mathrm{cm}$ and (b) relativistic runaway electrons in a homogeneous electric field of $12.5 \mathrm{kV} / \mathrm{cm}$ and thermal runaway electrons in the highly inhomogeneous electric field near the tip region of a $100 \mathrm{MV}$ lightning leader during the negative corona flash. The energy distributions are normalized so that the integration over electron energy from $1 \mathrm{MeV}$ to $100 \mathrm{MeV}$ yields unity. The results are obtained in air at ground level atmospheric density. The diamonds in Figure 6a show Monte Carlo simulation results obtained from Dwyer and Babich [2011, Figure 3], representing the energy distribution of electrons in RREAs driven by the electric field of $4.3 \mathrm{kV} / \mathrm{cm}$.

distribution is obtained after approximately 11 avalanche lengths, or $e$-folding times, and has already reached steady state. In spite of the noise in the energy distribution, it is clearly shown that over 6 orders of magnitude, the low-energy part $(<100 \mathrm{eV})$ is consistent with recently published full energy range model results [Colman et al., 2010] and BOLSIG+ calculations [Hagelaar and Pitchford, 2005]. We also note that the ratio between the number of electrons with energy below and above $1 \mathrm{MeV}$ is found to be 9365 , in agreement with the analytical calculation documented in Dwyer and Babich [2011].

Steady state electron energy distributions in RREAs, for the cases of homogeneous electric fields with magnitudes of $4.3 \mathrm{kV} / \mathrm{cm}$ and $12.5 \mathrm{kV} / \mathrm{cm}$, are presented in Figures $6 \mathrm{a}$ and $6 \mathrm{~b}$, respectively. The energy distributions are normalized so that the integration from $1 \mathrm{MeV}$ to $100 \mathrm{MeV}$ yields unity, following the assumption that the same number of electrons with energies above $1 \mathrm{MeV}$ is produced in the source of TGFs. Also presented in Figure 6a are Monte Carlo simulation results (diamonds) documented in Dwyer and Babich [2011, Figure 3], representing the energy distribution of electrons in RREAs driven by an electric field of $4.3 \mathrm{kV} / \mathrm{cm}$. For this electric field, the high-energy part of electron energy distribution shows excellent agreement between present work and results reported in Dwyer and Babich [2011, Figure 3]. We therefore estimate that our results on the electron energy distribution are validated over more than 15 orders of magnitude of the dynamic range. To our knowledge, such a result, over the full energy range of interest here, had never been obtained.

Unlike the case in Figure 3 (see discussion in section 3.1), energy distribution for electrons propagating under external electric fields, like in RREAs, is governed by the competition between the energy gained from electric fields and energy losses in collisions with neutral gas molecules, i.e., the dynamic friction force. The avalanche of relativistic runaway electrons is characterized by abundant generation of large quantities of secondary electrons over the full energy range. Secondary electrons with energies below $\sim 2 \mathrm{eV}$ are mostly trapped in this region because the applied electric field is insufficient to energize them to penetrate the vibrational barrier at $\sim 2 \mathrm{eV}$ (see Figure 3). Secondary electrons in the "valley" of the dynamic friction force (between $\sim 3 \mathrm{eV}$ and $\sim 15 \mathrm{eV}$ ) may be subject to an electric force that is capable of overcoming their energy losses in collisions with air molecules and therefore be accelerated to higher energies, depending on the strength of the driving electric field and the momentum of electrons. A driving electric field with a higher strength can surmount a larger dynamic friction force, corresponding to higher electron energies in the sharp increase between $\sim 4 \mathrm{eV}$ and $\sim 123 \mathrm{eV}$ (see Figure 3). Therefore, one sees from Figure $6 \mathrm{a}$ that, under a stronger electric field, more electrons are sustained in the energy range between $\sim 2 \mathrm{eV}$ and $\sim 15 \mathrm{eV}$. Also, in the high-energy part, the energy cutoff corresponding to the distribution obtained in $18.8 \mathrm{kV} / \mathrm{cm}$ is higher than that in $4.3 \mathrm{kV} / \mathrm{cm}$.

For the purpose of understanding the energy dependence of the capability of electrons in generating the upper excited states responsible for optical emissions from $2 \mathrm{PN}_{2}$ and $1 \mathrm{NN}_{2}^{+}$, the excitation frequencies 
Table 2. Intensity of Optical Emissions from $2 \mathrm{PN}_{2}$ (Column 3) and $1 \mathrm{NN}_{2}^{+}$(Column 4) in Rayleighs and Intensity Ratio Between $2 \mathrm{PN}_{2}$ and $1 \mathrm{NN}_{2}^{+}$(Column 5) in the Visible Range With Wavelengths Between $390 \mathrm{~nm}$ and $700 \mathrm{~nm}$ for Different Acceleration Processes (Column 1) With Different Characteristic Sizes (Column 2) Calculated at Ground Level

\begin{tabular}{lcccc} 
& Radius $(\mathrm{m})$ & $2 \mathrm{PN}_{2}(\mathrm{R})$ & $1 \mathrm{NN}_{2}^{+}(\mathrm{R})$ & $\frac{2 \mathrm{PN}_{2}}{1 \mathrm{NN}_{2}^{+}}$ \\
\hline RREA $(4.3 \mathrm{kV} / \mathrm{cm})$ & 1000 & $8.99 \times 10^{8}$ & $1.22 \times 10^{9}$ & 0.74 \\
RREA $(12.5 \mathrm{kV} / \mathrm{cm})$ & 1000 & $1.70 \times 10^{9}$ & $1.55 \times 10^{9}$ & 1.10 \\
RREA $(18.8 \mathrm{kV} / \mathrm{cm})$ & 1000 & $6.63 \times 10^{9}$ & $1.31 \times 10^{9}$ & 5.06 \\
Thermal runaway electrons & 50 & $8.28 \times 10^{11}$ & $5.23 \times 10^{11}$ & 1.58 \\
Streamer zone & 40 & $6.83 \times 10^{10}$ & $6.75 \times 10^{8}$ & 101.19 \\
\hline
\end{tabular}

are calculated using equation (3) and the energy distribution obtained in the present work and shown in Figure $5 a$. Figure $5 b$ presents the cumulative sum of the frequency for generating $\mathrm{N}_{2}\left(C^{3} \Pi_{u}\right)$ and $\mathrm{N}_{2}^{+}\left(B^{2} \Sigma_{u}^{+}\right)$in RREAs driven by an electric field of $70 \mathrm{Td}$. It is found that $50 \%$ of the upper excited states of $\mathrm{N}_{2}$ responsible for $2 \mathrm{PN}_{2}$ emissions is produced by electrons with energy below $12.75 \mathrm{eV}$ while $50 \%$ of the upper excited states of $\mathrm{N}_{2}^{+}$responsible for $1 \mathrm{NN}_{2}^{+}$emissions is produced by electrons with energy below $70.8 \mathrm{keV}$. This feature can be attributed to the fundamental difference in cross sections. The excitation cross section for generating $\mathrm{N}_{2}^{+}\left(B^{2} \Sigma_{u}^{+}\right)$rises rapidly above the threshold, attains a maximum value around $114 \mathrm{eV}$, drops off slowly to around $1 \mathrm{MeV}$, and then reaches a relativistic plateau above $1 \mathrm{MeV}$. Consequently, even though they are in minority, relativistic electrons efficiently ionize nitrogen molecules, resulting in comparable amount of $\mathrm{N}_{2}^{+}\left(B^{2} \Sigma_{u}^{+}\right)$when compared to that produced by low-energy electrons. In contrast, the cross section of $2 \mathrm{PN}_{2}$ falls off much faster after the peak and becomes negligible in the region above $1 \mathrm{keV}$, where ionization and elastic collisions dominate. As a result, optical emissions from $2 \mathrm{PN}_{2}$ are mainly produced by low-energy electrons. We note that since the electron impact excitation cross section for generating $\mathrm{N}_{2}\left(B^{3} \Pi_{g}\right)$ has a similar behavior as that of $2 \mathrm{PN}_{2}$, optical emissions from $1 \mathrm{PN}_{2}$ should also mainly come from low-energy electrons.

\subsubsection{Acceleration of Thermal Runaway Electrons in Lightning Leader Field}

Figure $6 \mathrm{~b}$ shows the full energy distribution for the ensemble of electrons accelerated in the highly inhomogeneous electric field around the tip region of a $100 \mathrm{MV}$ lightning leader during the negative corona flash stage. The electron energy distributions are normalized so that the integration from $1 \mathrm{MeV}$ to $100 \mathrm{MeV}$ yields unity. Due to the differences in geometry and magnitude between the homogeneous and inhomogeneous electric fields, a deviation from the RREA energy distribution is observed: a much smaller amount of electrons is generated and accumulated in the low-energy range and the high-energy tail does not follow the typical energy cutoff observed in RREAs.

\subsection{Optical Emissions}

Table 2 shows modeling results of optical emissions in the visible range with wavelengths between $390 \mathrm{~nm}$ and $700 \mathrm{~nm}$ from the following processes at ground level: RREAs, acceleration of thermal runaway electrons in the electric field produced by the $100 \mathrm{MV}$ lightning leader, and the streamer zone of the $100 \mathrm{MV}$ lightning leader. The columns 3 and 4 represent the maximum intensities of optical emissions from $2 \mathrm{PN}_{2}$ and $1 \mathrm{NN}_{2}^{+}$, respectively, during the listed processes. Column 5 shows the intensity ratio of $2 \mathrm{PN}_{2}$ to $1 \mathrm{NN}_{2}^{+}$ associated with the listed processes. Interestingly, both TGF production mechanisms produce considerable amount of optical emissions, with magnitudes comparable to those from the streamer zone associated with the $100 \mathrm{MV}$ lightning leader. The intensities of optical emissions from the streamer zone of the $100 \mathrm{MV}$ lightning leader are, respectively, $6.83 \times 10^{10} \mathrm{R}$ and $6.75 \times 10^{8} \mathrm{R}$ for $2 \mathrm{PN}_{2}$ and $1 \mathrm{NN}_{2}^{+}$. The intensity ratio of $2 \mathrm{PN}_{2}$ to $1 \mathrm{NN}_{2}^{+}$is $\sim 101.19$ as indicated in Liu et al. [2008].

The optical emissions from $2 \mathrm{PN}_{2}$ (column 3 of Table 2) associated with RREAs intensify with increases in driving electric fields. For instance, the intensities of optical emissions from $2 \mathrm{PN}_{2}$ corresponding to RREAs under electric fields of $4.3 \mathrm{kV} / \mathrm{cm}, 12.5 \mathrm{kV} / \mathrm{cm}$, and $18.8 \mathrm{kV} / \mathrm{cm}$ are, respectively, $8.99 \times 10^{8} \mathrm{R}, 1.70 \times 10^{9} \mathrm{R}$, and $6.63 \times 10^{9} \mathrm{R}$. However, optical emissions from $1 \mathrm{NN}_{2}^{+}$associated with RREAs do not indicate the same tendency. The intensities for $4.3 \mathrm{kV} / \mathrm{cm}, 12.5 \mathrm{kV} / \mathrm{cm}$, and $18.8 \mathrm{kV} / \mathrm{cm}$ are, respectively, $1.22 \times 10^{9} \mathrm{R}$, $1.55 \times 10^{9} \mathrm{R}$, and $1.31 \times 10^{9} \mathrm{R}$. To understand how these characteristic tendencies in optical output stem from the differences in electron energy distributions, it is helpful to look at the comparison presented in Figure 6a. As the homogeneous electric field increases from $4.3 \mathrm{kV} / \mathrm{cm}$ to $18.8 \mathrm{kV} / \mathrm{cm}$, a larger population of 
electrons is generated and sustained in the energy region from $\sim 11 \mathrm{eV}$ (the threshold energy of producing $\mathrm{N}_{2}\left(C^{3} \Pi_{u}\right)$ ) to $\sim 15 \mathrm{eV}$, which plays a critical role in generating optical emissions from $2 \mathrm{PN}_{2}$ (see Figure $5 \mathrm{~b}$ and discussion in section 3.2.1). Therefore, a stronger driving electric field should demonstrate more intense optical emissions from $2 \mathrm{PN}_{2}$ during RREAs as evident in Table 2. Contrary to $2 \mathrm{PN}_{2}$, optical emissions from $1 \mathrm{NN}_{2}^{+}$can be as efficiently produced by high-energy electrons as by low-energy ones (see Figure $5 \mathrm{~b}$ and discussion in section 3.2.1). Considering that the energy distribution of electrons accelerating in $18.8 \mathrm{kV} / \mathrm{cm}$ only slightly differs from that of $4.3 \mathrm{kV} / \mathrm{cm}$ in the energy region above $\sim 20 \mathrm{eV}$ (the threshold energy of producing $\mathrm{N}_{2}^{+}\left(B^{2} \Sigma_{u}^{+}\right)$), optical emissions from $1 \mathrm{NN}_{2}^{+}$should not change considerably, as also evident in Table 2.

The two TGF production mechanisms exhibit distinct optical features. First, when compared to RREA processes, optical emissions associated with the acceleration of thermal runaway electrons in lightning leader fields are more intense, with $8.28 \times 10^{11} \mathrm{R}$ from $2 \mathrm{PN}_{2}$, and $5.23 \times 10^{11} \mathrm{R}$ from $1 \mathrm{NN}_{2}^{+}$. This is primarily because the production of TGFs by thermal runaway electrons is naturally confined to a relatively compact region in the vicinity of negative leader tip [e.g., Xu et al., 2012; Celestin et al., 2012]. Second, the intensity ratios of $2 \mathrm{PN}_{2}$ to $1 \mathrm{NN}_{2}^{+}$are different between the two production mechanisms. For example, the intensity ratios between $2 \mathrm{PN}_{2}$ and $1 \mathrm{NN}_{2}^{+}$are, respectively, $0.74,1.10$, and 5.06 for RREAs driven by homogeneous electric fields of $4.3 \mathrm{kV} / \mathrm{cm}, 12.5 \mathrm{kV} / \mathrm{cm}$, and $18.8 \mathrm{kV} / \mathrm{cm}$ while this ratio is 1.58 for thermal runaway electrons produced and accelerated in the vicinity of the tip of a $100 \mathrm{MV}$ lightning leader. This feature is also a reflection of the inherent differences in electron energy distributions. In the case of RREAs, as discussed in the previous paragraph, a stronger driving electric field enables more intense optical emissions from $2 \mathrm{PN}_{2}$ while producing similar levels of emissions from $1 \mathrm{NN}_{2}^{+}$. For this reason, one observes in Table 2 that the intensity ratio between $2 \mathrm{PN}_{2}$ and $1 \mathrm{NN}_{2}^{+}$in RREAs becomes larger with the increase in driving electric fields. Nevertheless, as illustrated in Figure $6 \mathrm{~b}$, the electron energy distributions are similar in the energy regions critical for optical emissions from $2 \mathrm{PN}_{2}$ and $1 \mathrm{NN}_{2}^{+}$between the processes of thermal runaway electrons accelerating in a $100 \mathrm{MV}$ lightning leader field and RREAs under an electric field of $12.5 \mathrm{kV} / \mathrm{cm}$. Thus, the intensity ratios of $2 \mathrm{PN}_{2}$ to $1 \mathrm{NN}_{2}^{+}$are close between these two processes as presented in Table 2 .

\section{Discussion}

\subsection{Fluorescence Efficiency}

For the sake of comparison with previously published results [Dwyer et al., 2013], we have calculated the fluorescence efficiency without collisional quenching corresponding to the steady state of RREAs for $2 \mathrm{PN}_{2}$ and $1 \mathrm{NN}_{2}^{+}$using:

$$
\epsilon_{k}=\frac{v_{k} \sum_{\lambda} E_{\lambda} l_{k}(\lambda)}{\int F_{d}(\varepsilon) v(\varepsilon) f(\varepsilon) \mathrm{d} \varepsilon}
$$

where $E_{\lambda}$ is the energy of fluorescence photons with a wavelength of $\lambda$ defined as $E_{\lambda}=h c / \lambda$, where $h$ is the Planck's constant and $c$ is the speed of light in free space, $I_{k}(\lambda)$ is the intensity distribution of optical emissions from either $2 \mathrm{PN}_{2}$ or $1 \mathrm{NN}_{2}^{+}$obtained from Vallance Jones [1974] and is normalized so that $\sum_{\lambda} I_{k}(\lambda)=1$, and $F_{d}(\varepsilon)$ is the dynamic friction force due to collisions with air molecules that electrons with an energy of $\varepsilon$ experience at ground level (see Figure 3). The summation in the numerator is taken over wavelengths of major emission bands from $2 \mathrm{PN}_{2}$ or $1 \mathrm{NN}_{2}^{+}$.

It is found that the fluorescence efficiencies of $2 \mathrm{PN}_{2}$ for RREAs driven by electric fields of $4.3 \mathrm{kV} / \mathrm{cm}$, $12.5 \mathrm{kV} / \mathrm{cm}$, and $18.8 \mathrm{kV} / \mathrm{cm}$, in the visible range of 390-700 nm without collisional quenching, are $0.0146 \%$, $0.00287 \%$, and $0.0208 \%$, respectively. Fluorescence efficiencies of $1 \mathrm{NN}_{2}^{+}$, in the same wavelength range without collisional quenching, are $0.213 \%, 0.0283 \%$, and $0.0443 \%$ for RREAs driven by electric fields of $4.3 \mathrm{kV} / \mathrm{cm}, 12.5 \mathrm{kV} / \mathrm{cm}$, and $18.8 \mathrm{kV} / \mathrm{cm}$, respectively. Based on these calculations, fluorescence efficiency in RREAs can vary notably for driving electric fields with different strengths.

More intense driving electric fields do not necessarily facilitate the associated fluorescence efficiency, even if they result in more energy deposition. In RREAs, energy deposition and fluorescence emissions are caused by electrons in different energy regions. To illustrate this discussion, we have verified that, for the three electric fields studied in the present work, most of the energy deposited via collisions with air molecules is from the large ensemble of electrons in the energy region below $\sim 4 \mathrm{eV}$ while optical emissions from $2 \mathrm{PN}_{2}$ are mostly produced by electrons with energies between $\sim 11 \mathrm{eV}$ and $100 \mathrm{eV}$. 
As the driving electric field changes from $4.3 \mathrm{kV} / \mathrm{cm}$ to $12.5 \mathrm{kV} / \mathrm{cm}$, although secondary electrons in the energy region between $\sim 11 \mathrm{eV}$ and $100 \mathrm{eV}$ are able to excite more $\mathrm{N}_{2}\left(C^{3} \Pi_{u}\right)$ and release more energy to generate fluorescence photons from $2 \mathrm{PN}_{2}$, secondary electrons in the energy region below $\sim 4 \mathrm{eV}$ much more efficiently deposit their kinetic energy into air molecules through vibrational and rotational excitations. As a consequence, the fluorescence efficiency for $2 \mathrm{PN}_{2}$ decreases. However, when the driving electric field further increases to $18.8 \mathrm{kV} / \mathrm{cm}$, a much larger population of secondary electrons can be accelerated to and maintained in the energy region that is critical for $2 \mathrm{PN}_{2}$ and, therefore, an enhancement in fluorescence efficiency is observed. Similarly, fluorescence efficiency for $1 \mathrm{NN}_{2}^{+}$in RREAs is also highly dependent on the strength of the driving electric field.

Moreover, extensive air showers have also been studied through modeling of the deceleration of $1 \mathrm{MeV}$ electrons [e.g., Lafebre et al., 2009] in the atmosphere. Using the same model as discussed in the present paper, we have estimated the fluorescence efficiencies of $1 \mathrm{MeV}$ electrons decelerating through air. It is interesting to note that the fluorescence efficiencies for $2 \mathrm{PN}_{2}$ and $1 \mathrm{NN}_{2}^{+}$, between 390 and $700 \mathrm{~nm}$ and without collisional quenching, are found to be $0.0405 \%$ and $0.554 \%$, respectively, that are very close to the values reported and used in Dwyer et al. [2013]. We note that the calculations of Dwyer et al. [2013] were based on experimentally measured fluorescence efficiency for $0-0$ bands of $2 \mathrm{PN}_{2}$ and $1 \mathrm{NN}_{2}^{+}$ [Keilhauer et al., 2006].

Additionally, we have estimated the amount of fluorescence photons that can be generated per runaway electron per meter in the wavelength range between 300 and $430 \mathrm{~nm}$ corresponding to the steady state of RREAs. This wavelength range is chosen in order to cover major emission bands of $2 \mathrm{PN}_{2}$ and $1 \mathrm{NN}_{2}^{+}$. The values are approximately $6.2,10.7$, and $38.3 \mathrm{~m}^{-1}$ for RREAs driven by electric fields of $4.3 \mathrm{kV} / \mathrm{cm}, 12.5 \mathrm{kV} / \mathrm{cm}$, and $18.8 \mathrm{kV} / \mathrm{cm}$ at ground pressure, respectively. This shows that the stronger the sustaining electric fields, the more intense the associated fluorescence emissions. Note that this is consistent with the transition in electron energy distributions (i.e., enhancement in low-energy part with increasing applied electric field) presented in Figure 6a.

Fluorescence efficiency, in the study of air showers, describes a process in which primary electrons eventually deposit all their initial kinetic energy into air molecules of the Earth's atmosphere, which release it through emissions of fluorescence and collisional deexcitation. However, in TGFs, the acceleration of electrons and the subsequent deposition of energy into air molecules are supported by external electric fields. The amount of energy that is converted into fluorescence emissions mainly comes from that acquired from the driving external electric field. Therefore, we suggest that fluorescence efficiency in extensive air showers is not conceptually identical to that in TGFs. Also, the electron energy distribution obtained from the deceleration process in extensive air showers can be substantially different from that in TGFs. Due to the fact that the capability of electrons in generating fluorescence photons is strongly energy-dependent (see Figure $5 \mathrm{~b}$ and discussion in section 3.2.1), fluorescence efficiency measured in extensive air showers is different from that in TGFs.

\subsection{Scaling of Optical Emissions}

Modeling results of optical emissions from the acceleration of thermal runaway electrons in lightning leader field and the associated streamer zone (Table 2 ) are obtained at ground level air density and can be applied to the source altitudes of TGFs using similarity scaling. As indicated in equation (1), the intensity of optical emissions is closely related to the number density of excited species $n_{k}$. For the two optical band systems considered in the present work, i.e., $2 \mathrm{PN}_{2}$ and $1 \mathrm{NN}_{2}^{+}, n_{k}$ is populated by electron impact excitations (the term $v_{k} n_{e}$ in equation (2)) and depopulated by spontaneous emissions (the term $A_{k}$ in $\tau_{k}$ ) and collisional quenching processes with air molecules (the terms $\alpha_{1} N_{\mathrm{N}_{2}}$ and $\alpha_{2} N_{\mathrm{O}_{2}}$ in $\tau_{k}$ ). At or below the source altitudes of TGFs [e.g., Dwyer and Smith, 2005; Xu et al., 2012], collisional quenching processes dominate over spontaneous emissions and, in the steady state, $n_{k}$ is obtained by the balance between direct excitations and collisional quenching. Since both collisional quenching $\left(\alpha_{1} N_{\mathrm{N}_{2}}+\alpha_{2} N_{\mathrm{O}_{2}}\right)$ and electron impact excitation frequency $\left(v_{k}\right)$ scale as the number density of air molecules $(\sim N), n_{k}$ scales as the number density of electrons $n_{e}$.

The total number of energetic electrons emitted from a lightning leader is invariant of air pressure [Moss et al., 2006]. The characteristic radial dimension of the acceleration of thermal runaway electrons in the lightning leader field is defined as the region in which thermal runaway electrons accelerate and efficiently gain energy. This condition is fulfilled if the electric force exerted on thermal runaway electrons $q_{e} E$ 
exceeds the average friction force due to collisions with air molecules $q_{e} E_{t}$, where $q_{e}$ is the electron elementary charge, $E$ is the inhomogeneous electric field produced around lightning leader tips, and $E_{\mathrm{t}}$ is the relativistic runaway threshold field and its value at ground level atmospheric density is $\sim 2.8 \mathrm{kV} / \mathrm{cm}$ [e.g., Dwyer et al., 2012]. As suggested by Celestin et al. [2012], the highly inhomogeneous electric field $E$ produced by high-potential lightning leaders can be approximated by an $A / r$ function, where $A=0.04 E_{0} I$ is a fitting parameter and $r$ is the distance from the leader tip. Since $r$ varies inversely as $E$ and $E_{\mathrm{t}}$ scales proportionally with the density of the neutral atmosphere, i.e., $E_{\mathrm{t}} \sim N$, the characteristic radial dimension representing the acceleration of thermal runaway electrons in lightning leader field scales as $\sim 1 / \mathrm{N}$. Therefore, the number density of energetic electrons responsible for producing $\mathrm{N}_{2}\left(C^{3} \Pi_{u}\right)$ or $\mathrm{N}_{2}^{+}\left(B^{2} \Sigma_{u}^{+}\right)$ scales as $\sim N^{3}$. Furthermore, considering that the intensity of optical emissions in Rayleighs is obtained by integrating over the line of sight, which scales as $\sim 1 / N$, and remembering that $n_{k}$ scales in the same way as $n_{e^{\prime}}$ one sees that the cross-sectional intensity of optical emissions (number of photons per unit area) produced during the acceleration of thermal runaway electrons in lightning leader fields scales as $\sim N^{2}$. For example, the characteristic radius for the acceleration process of thermal runaway electrons in the $100 \mathrm{MV}$ lightning leader case is $50 \mathrm{~m}$ at ground level. However, this quantity at an altitude of $12 \mathrm{~km}$, corresponding to the source altitude of TGFs [e.g., Xu et al., 2012], would be $\sim 211 \mathrm{~m}$. The intensity of $2 \mathrm{PN}_{2}$ is $8.28 \times 10^{11} \mathrm{R}$ at ground level and would be reduced to $\sim 4.63 \times 10^{10} \mathrm{R}$ at $12 \mathrm{~km}$ altitude.

As for the streamer zone associated with the $100 \mathrm{MV}$ lightning leader, its radius $R_{\mathrm{sz}}^{-}$is inversely proportional to $E_{s}^{-}$, which scales as $\sim N$. Thus, $R_{\mathrm{sz}}$ scales as $\sim 1 / N$. For the same reason, the total amount of electric charge contained in the streamer zone $Q_{s}$ also scales as $\sim 1 / N$ (see equation (4)). Because the streamer electron density scales as $\sim N^{2}$ and the length scale of streamers scales as $\sim 1 / N$ [e.g., Liu and Pasko, 2004], the average electric charge carried by a streamer $q_{s}$ scales as $\sim 1 / N$. Since both $Q_{s}$ and $q_{s}$ scale as $\sim 1 / N$, the number of streamers constituting a streamer zone, which follows the relation $N_{s}=Q_{s} / q_{s}$, remains the same with respect to air pressure/altitude. In a single streamer, $n_{k}$ scales as the streamer electron density, $\sim N^{2}$. As a result, by integrating over the line of sight, the total cross-sectional intensity of optical emissions from the streamer zone scales as $\sim N$.

\subsection{Optical Emissions Associated With TGFs}

Different driving electric fields can lead to different acceleration and multiplication processes undergone by runaway electrons, different energy distributions obtained, and thus different capabilities in generating optical emissions. In RREA mechanism, relativistic runaway electrons harvest energy from the homogeneous electric field and gradually build up a $\sim 7 \mathrm{MeV}$ high-energy cutoff while producing a large ensemble of low-energy electrons. Reciprocally, as demonstrated in Celestin et al. [2012], the intense inhomogeneous electric field naturally present in compact regions around negative lightning leader tips during stepping processes can accelerate thermal runaway electrons to high energy over much shorter distances, corresponding to much less low-energy electrons generated and a significantly different high-energy cutoff. A direct consequence of these differences is that the optical output associated with these two mechanisms is different. For example, typical magnitude of the large spatial scale electric field measured within thunderstorms is lower than $4.3 \mathrm{kV} / \mathrm{cm}$ [e.g., Marshall et al., 2001], the optical output from RREA processes driven by such field would be much weaker than that resulting from the acceleration process of thermal runaway electrons in lightning leader fields (see Table 2). In addition, the intensity ratio between $2 \mathrm{PN}_{2}$ and $1 \mathrm{NN}_{2}^{+}$would be 1 order of magnitude smaller than that of the acceleration and multiplication processes of thermal runaway electrons in lightning leader fields.

TGF events are most likely accompanied with detectable levels of optical emissions for both existing models as suggested by modeling results (see Table 2). These optical emissions would be dominantly blue, or purplish blue (as previously noted by Dwyer et al. [2013]). Moreover, using modeling results of optical emissions produced during the acceleration of thermal runaway electrons in lightning leader fields (see Table 2) and considering that this process typically lasts from a few tens of microseconds to a fraction of millisecond, we estimate that the total amount of optical energy radiated in the visible wavelength range in this scenario would be on the order of a few tens of joules. It is comparable to that theoretically produced during relativistic feedback discharges [Dwyer et al., 2013] but much smaller than that of normal lightning [Uman, 2001]. Further comparisons between modeling results and ground-based or space-based observations would require the transport of optical photons through the atmosphere to be taken into account. This is especially important in view of the future space missions ASIM (ESA) and TARANIS (CNES) 
that will detect TGFs from the nadir direction in association with photometric measurements. Furthermore, the temperature and humidity dependence of air fluorescence emissions have been recently observed [e.g., Ave et al., 2008] and this effect should be accounted for to ensure accurate interpretation of optical observations.

The theory of TGFs produced by thermal runaway electrons initiated during negative corona flashes may introduce a chronological order between the optical emissions from molecules excited by thermal runaway electrons and their by-products, and those from the streamer zone. Indeed, one can expect that most thermal runaway electrons and their corresponding optical emissions would be produced during the early establishment of the streamer zone, the transient stage of negative corona flashes. According to the discussion in Moss et al. [2006], the duration of the negative corona flash can be estimated from the speed of streamers and the size of the eventual streamer zone. Considering that streamers, on average, have speeds on the order of $\sim 5 \times 10^{6} \mathrm{~m} / \mathrm{s}$ [e.g., Stenbaek-Nielsen et al., 2013] and the typical size of the streamer zone associated with high-potential lightning leaders in +ICs is in the range 50-600 m [e.g., Winn et al., 2011], the corresponding negative corona flashes should have a duration in the range 10-200 $\mu$ s. However, streamer zones could last for much longer time, typically in the range from $100 \mu \mathrm{s}$ to $1 \mathrm{~ms}$ in $+\mathrm{ICs}$ [e.g., Marshall et al., 2013; Lu et al., 2011]. The lightning leaders in -CGs can emit intense bursts of X-rays during the stepping process [e.g., Dwyer et al., 2005]. It is possible that fluorescence light is also observable around the tip of these leaders.

Even if difficult to observe, optical emissions from molecules excited by thermal runaway electrons accelerating in lightning leader fields are temporally and spatially separated from those normally produced by lightning leaders. Optical emissions associated with the acceleration process of thermal runaway electrons in lightning leader fields would be produced right after the establishment of a new leader step, while the lightning leader would emit light during the connection of the new step. Additionally, since thermal runaway electrons gain energy from the electric field produced in the vicinity of lightning leader tips, fluorescence photons caused by thermal runaway electrons would be emitted from a compact region in front of lightning leaders. Moreover, considering that electrons involved in lightning discharges and those produced by thermal runaway electrons can follow significantly different energy distributions, it is conceivable that the intensity ratio of optical emissions from $2 \mathrm{PN}_{2}$ and $1 \mathrm{NN}_{2}^{+}$can be considerably different between the two processes. Finally, the production of optical emissions by RREAs sustained by large-scale thunderstorm electric fields is not necessarily associated with stepping lightning leaders and could therefore be observed in the absence of light from lightning discharges.

\section{Conclusions}

Principal contributions of this paper can be summarized as follows:

1. Using a full energy relativistic Monte Carlo model, we have investigated the fluorescence emissions from air excited by energetic electrons at high pressures. Simulation results, including the morphological features of fluorescence emissions generated by a continuous beam of $50 \mathrm{keV}$ electrons and the fluorescence efficiency for the $2 \mathrm{PN}_{2}$ and $1 \mathrm{NN}_{2}^{+}$band systems produced by $850 \mathrm{keV}$ electrons, are consistent with laboratory observations [Davidson and O'Neil, 1964; Nagano et al., 2004]. The importance of the dynamic friction force in characterizing electron energy distribution has been emphasized.

2. We have studied the characteristics of electron energy distributions over the full energy range for two TGF production mechanisms: RREAs and production of thermal runaway electrons during the negative corona flash stages of lightning leaders. The simulated energy distribution of RREAs is in good agreement with results presented in e.g., Dwyer and Babich [2011] and Dwyer et al. [2012] in the high-energy region and BOLSIG+ calculation [Hagelaar and Pitchford, 2005] and simulation results in Colman et al. [2010] in the lower energy part. Furthermore, we have emphasized that the capability of RREA electrons in generating the upper excited states responsible for optical emissions from $2 \mathrm{PN}_{2}$ and $1 \mathrm{NN}_{2}^{+}$is strongly energy dependent. It has also been illustrated how RREAs and the acceleration of thermal runaway electrons in the electric field produced by high-potential lightning leader lead to inherently different electron energy distributions.

3. From the knowledge of the electron energy distribution, we have quantified the optical emissions that are possibly generated during the production of TGFs. Modeling results indicate that TGFs are most likely accompanied with detectable levels of optical emissions. However, optical emissions produced during 


\section{Acknowledgments}

This research was supported by the NSF grants AGS-1106779 and AGS-0741589 to Penn State University. Sebastien Celestin's research was supported by the French space agency (CNES). Some of the simulation results presented in this paper have been obtained using the cluster at the Centre de Calcul Scientifique en région Centre (CCSC). All data used in this paper are directly available after a request is made to authors W.X. (wxx5015@psu.edu), S.C. (sebastien.celestin@cnrs-orleans.fr), or V.P.P. (vpasko@psu.edu).

Michael Liemohn thanks Gerald Fishman and another reviewer for their assistance in evaluating this paper. the acceleration of thermal runaway electrons in lightning leader fields are more intense than those associated with RREAs. The intensity ratio between $2 \mathrm{PN}_{2}$ and $1 \mathrm{NN}_{2}^{+}$is intrinsically different between these two TGF production mechanisms.

4. Calculations of fluorescence efficiencies corresponding to air showers and the steady state of RREAs have shown that, although broadly used in the study of air showers to identify the energy of primary particles, these fluorescence efficiencies are not appropriate for the study of optical emissions from TGF sources.

5. Optical emissions, appearing as the low-energy signature of the underlying electron acceleration mechanism, are a good probe to point to key ingredients in TGF production. The distinct optical features described in the present work can be used to constrain and validate existing TGF models. Moreover, since TGFs are closely associated with the initial development of +IC flashes [e.g., Marshall et al., 2013], measurements of optical emissions also have important implications for studies of the initial breakdown stage of lightning flashes.

\section{References}

Adachi, T., H. Fukunishi, Y. Takahashi, Y. Hiraki, R.-R. Hsu, H.-T. Su, A. B. Chen, S. B. Mende, H. U. Frey, and L. C. Lee (2006), Electric field transition between the diffuse and streamer regions of sprites estimated from ISUAL/array photometer measurements, Geophys. Res. Lett., 33, L17803, doi:10.1029/2006GL026495.

Ave, M., et al. (2008), Temperature and humidity dependence of air fluorescence yield measured by AIRFLY, Nucl. Instr. and Meth. A., 597, 50-54, doi:10.1016/j.nima.2008.08.050.

Babaeva, N. Y., and G. V. Naidis (1997), Dynamics of positive and negative streamers in air in weak uniform electric fields, IEEE Trans. Plasma Sci., 25, 375-379, doi:10.1109/27.602514.

Babich, L. P., E. N. Donskoy, R. I. II'Kaev, I. M. Kutsyk, and R. A. Roussel-Dupre (2004), Fundamental parameters of a relativistic runaway electron avalanche in air, Plasma Phys. Rep., 30, 616-624, doi:10.1134/1.1778437.

Balanis, C. A. (1989), Advanced Engineering Electromagnetics, John Wiley, New York.

Bazelyan, E. M., and Y. P. Raizer (2000), Lightning Physics and Lightning Protection, Inst. of Phys., Bristol, Pa.

Briggs, M. S., et al. (2010), First results on terrestrial gamma ray flashes from the Fermi Gamma-ray Burst Monitor, J. Geophys. Res., 115, A07323, doi:10.1029/2009JA015242.

Bunner, A. N. (1967), Cosmic ray detection by atmospheric fluorescence, PhD thesis, Cornell University, Ithaca, N. Y

Celestin, S., and V. P. Pasko (2010a), Effects of spatial non-uniformity of streamer discharges on spectroscopic diagnostics of peak electric fields in transient luminous events, Geophys. Res. Lett., 37, L07804, doi:10.1029/2010GL042675.

Celestin, S., and V. P. Pasko (2010b), Soft collisions in relativistic runaway electron avalanches, J. Phys. D: Appl. Phys., 43, 315206, doi:10.1088/0022-3727/43/31/315206.

Celestin, S., and V. P. Pasko (2011), Energy and fluxes of thermal runaway electrons produced by exponential growth of streamers during the stepping of lightning leaders and in transient luminous events, J. Geophys. Res., 116, A03315, doi:10.1029/2010JA016260.

Celestin, S., W. Xu, and V. P. Pasko (2012), Terrestrial gamma ray flashes with energies up to $100 \mathrm{MeV}$ produced by nonequilibrium acceleration of electrons in lightning, J. Geophys. Res., 117, A05315, doi:10.1029/2012JA017535.

Chamberlain, J. W. (1978), Theory of Planetary Atmospheres, Academic Press, San Diego, Calif.

Colman, J. J., R. A. Roussel-Dupré, and L. Triplett (2010), Temporally self-similar electron distribution functions in atmospheric breakdown: The thermal runaway regime, J. Geophys. Res., 115, A00E16, doi:10.1029/2009JA014509.

Davidson, G., and R. O'Neil (1964), Optical radiation from nitrogen and air at high pressure excited by energetic electrons, J. Chem. Phys., 41, 3946-3955, doi:10.1063/1.1725841.

Dwyer, J. R. (2007), Relativistic breakdown in planetary atmospheres, Phys. Plasmas, 14, 042901, doi:10.1063/1.2709652.

Dwyer, J. R. (2008), Source mechanisms of terrestrial gamma-ray flashes, J. Geophys. Res., 113, D10103, doi:10.1029/2007JD009248.

Dwyer, J. R., and L. P. Babich (2011), Low-energy electron production by relativistic runaway electron avalanches in air, J. Geophys. Res., 116, A09301, doi:10.1029/2011JA016494.

Dwyer, J. R., and D. M. Smith (2005), A comparison between Monte Carlo simulations of runaway breakdown and terrestrial gamma-ray flash observations, Geophys. Res. Lett., 32, L22804, doi:10.1029/2005GL023848.

Dwyer, J. R., et al. (2005), X-ray bursts associated with leader steps in cloud-to-ground lightning, Geophys. Res. Lett., 32, L01803, doi:10.1029/2004GL021782.

Dwyer, J. R., D. M. Smith, and S. A. Cummer (2012), High-energy atmospheric physics: Terrestrial gamma-ray flashes and related phenomena, Space Sci. Rev., 173, 133-196, doi:10.1007/s11214-012-9894-0.

Dwyer, J. R., N. Y. Liu, and H. K. Rassoul (2013), Properties of the thundercloud discharges responsible for terrestrial gamma-ray flashes, Geophys. Res. Lett., 40, 4067-4073, doi:10.1002/grl.50742.

Fishman, G. J., et al. (1994), Discovery of intense gamma-ray flashes of atmospheric origin, Science, 264(5163), 1313-1316.

Fishman, G. J., et al. (2011), Temporal properties of the terrestrial gamma-ray flashes from the Gamma-Ray Burst Monitor on the Fermi Observatory, J. Geophys. Res., 116, A07304, doi:10.1029/2010JA016084.

Gurevich, A. V. (1961), On the theory of runaway electrons, Sov. Phys. JETP, 12, 904-912.

Gurevich, A. V., G. M. Milikh, and R. A. Roussel-Dupré (1992), Runaway electron mechanism of air breakdown and preconditioning during a thunderstorm, Phys. Lett. A, 165(5-6), 463-468, doi:10.1016/0375-9601(92)90348-P.

Hagelaar, G. J. M., and L. C. Pitchford (2005), Solving the Boltzmann equation to obtain electron transport coefficients and rate coefficients for fluid models, Plasma Sources Sci. Technol., 14, 722-733, doi:10.1088/0963-0252/14/4/011.

Keilhauer, B., J. Blümer, R. Engel, and H. O. Klages (2006), Impact of varying atmospheric profiles on extensive air shower observation: Fluorescence light emission and energy reconstruction, Astropart. Phys., 25, 259-268, doi:10.1016/j.astropartphys.2006.02.005.

Kim, Y.-K., J. P. Santos, and F. Parente (2000), Extension of the binary-encounter-dipole model to relativistic incident electrons, Phys. Rev. A, 62, 052710, doi:10.1103/PhysRevA.62.052710.

Kossyi, I. A., A. Y. Kostinsky, A. A. Matveyev, and V. P. Silakov (1992), Kinetic scheme of the non-equilibrium discharge in nitrogen-oxygen mixtures, Plasma Sources Sci. Technol., 1, 207-220, doi:10.1088/0963-0252/1/3/011. 
Kuo, C.-L., R. R. Hsu, A. B. Chen, H. T. Su, L. C. Lee, S. B. Mende, H. U. Frey, H. Fukunishi, and Y. Takahashi (2005), Electric fields and electron energies inferred from the ISUAL recorded sprites, Geophys. Res. Lett., 32, L19103, doi:10.1029/2005GL023389.

Kuo, C.-L., et al. (2009), Discharge processes, electric field, and electron energy in ISUAL-recorded gigantic jets, J. Geophys. Res., 114, A04314, doi:10.1029/2008JA013791.

Lafebre, S., R. Engel, H. Falcke, J. Hörandel, T. Huege, J. Kuijpers, and R. Ulrich (2009), Universality of electron-positron distributions in extensive air showers, Astropart. Phys., 31, 243-254, doi:10.1016/j.astropartphys.2009.02.002.

Liu, N., and V. P. Pasko (2004), Effects of photoionization on propagation and branching of positive and negative streamers in sprites, J. Geophys. Res., 109, A04301, doi:10.1029/2003JA010064.

Liu, N., et al. (2006), Comparison of results from sprite streamer modeling with spectrophotometric measurements by ISUAL instrument on FORMOSAT-2 satellite, Geophys. Res. Lett., 33, L01101, doi:10.1029/2005GL024243.

Liu, N. Y., S. Célestin, A. Bourdon, V. P. Pasko, P. Ségur, and E. Marode (2008), Photoionization and optical emission effects of positive streamers in air at ground pressure, IEEE Trans. Plasma Sci., 36(4), 942-943.

Lu, G., R. J. Blakeslee, J. Li, D. M. Smith, X.-M. Shao, E. W. McCaul, D. E. Buechler, H. J. Christian, J. M. Hall, and S. A. Cummer (2010), Lightning mapping observation of a terrestrial gamma-ray flash, Geophys. Res. Lett., 37, L11806, doi:10.1029/2010GL043494.

Lu, G., S. A. Cummer, J. Li, F. Han, D. M. Smith, and B. W. Grefenstette (2011), Characteristics of broadband lightning emissions associated with terrestrial gamma ray flashes, J. Geophys. Res., 116, A03316, doi:10.1029/2010JA016141.

Marisaldi, M., et al. (2010), Detection of terrestrial gamma ray flashes up to $40 \mathrm{MeV}$ by the AGILE satellite, J. Geophys. Res., 115, A00E13, doi:10.1029/2009JA014502.

Marshall, T., M. Stolzenburg, S. Karunarathne, S. Cummer, G. Lu, H.-D. Betz, M. Briggs, V. Connaughton, and S. Xiong (2013), Initial breakdown pulses in intracloud lightning flashes and their relation to terrestrial gamma ray flashes, J. Geophys. Res. Atmos., 118, 10,907-10,925, doi:10.1002/jgrd.50866.

Marshall, T. C., M. Stolzenburg, W. D. Rust, E. R. Williams, and R. Boldi (2001), Positive charge in the stratiform cloud of a mesoscale convective system, J. Geophys. Res., 106(D1), 1157-1163, doi:10.1029/2000JD900625.

Mitchell, K. B. (1970), Fluorescence efficiencies and collisional deactivation rates for $\mathrm{N}_{2}$ and $\mathrm{N}_{2}^{+}$bands excited by soft $\mathrm{X}$ rays, J. Chem. Phys., 53, 1795, doi:10.1063/1.1674257.

Morrill, J. S., and W. M. Benesch (1996), Auroral $\mathrm{N}_{2}$ emissions and the effect of collisional processes on $\mathrm{N}_{2}$ triplet state vibrational populations, J. Geophys. Res., 101(A1), 261-274, doi:10.1029/95JA02835.

Moss, G. D., V. P. Pasko, N. Liu, and G. Veronis (2006), Monte Carlo model for analysis of thermal runaway electrons in streamer tips in transient luminous events and streamer zones of lightning leaders, J. Geophys. Res., 111, A02307, doi:10.1029/2005JA011350.

Nagano, M., K. Kobayakawa, N. Sakaki, and K. Ando (2004), New measurement on photon yields from air and the application to the energy estimation of primary cosmic rays, Astropart. Phys., 22, 235-248, doi:10.1016/j.astropartphys.2004.08.002.

Østgaard, N., T. Gjesteland, B. E. Carlson, A. B. Collier, S. A. Cummer, G. Lu, and H. J. Christian (2013), Simultaneous observations of optical lightning and terrestrial gamma ray flash from space, Geophys. Res. Lett., 40, 2423-2426, doi:10.1002/grl.50466.

Pancheshnyi, S. V., S. M. Starikovskaia, and A. Y. Starikovskii (1998), Measurements of rate constants of the $N_{2}\left(C^{3} \Pi_{u}, v^{\prime}=0\right)$ and $\mathrm{N}_{2}^{+}\left(B^{2} \Sigma_{u}^{+}, v^{\prime}=0\right)$ deactivation by $\mathrm{N}_{2}, \mathrm{O}_{2}, \mathrm{H}_{2}, \mathrm{CO}$ and $\mathrm{H}_{2} \mathrm{O}$ molecules in afterglow of the nanosecond discharge, Chem. Phys. Lett., 294, 523-527, doi:10.1016/S0009-2614(98)00879-3.

Pasko, V. P. (2014), Electrostatic modeling of intracloud stepped leader electric fields and mechanisms of terrestrial gamma ray flashes, Geophys. Res. Lett., 41, 179-185, doi:10.1002/2013GL058983.

Piper, L. G. (1988), State-to-state $\mathrm{N}_{2}\left(A^{3} \Sigma_{u}^{+}\right)$energy pooling reactions. II. The formation and quenching of $\mathrm{N}_{2}\left(B^{3} \Pi_{g}, v^{\prime}=1-12\right), J . C h e m$. Phys., 88, 6911, doi:10.1063/1.454388.

Rakov, V. A., and M. A. Uman (2003), Lightning: Physics and Effects, Cambridge Univ. Press, Cambridge, U. K.

Shao, X.-M., T. Hamlin, and D. M. Smith (2010), A closer examination of terrestrial gamma-ray flash-related lightning processes, J. Geophys. Res., 115, A00E30, doi:10.1029/2009JA014835.

Sipler, D. P., and M. A. Biondi (1972), Measurements of $O\left({ }^{1} D\right)$ quenching rates in the $F$ region, J. Geophys. Res., 77, 6202-6212.

Smith, D. M., L. I. Lopez, R. P. Lin, and C. P. Barrington-Leigh (2005), Terrestrial gamma-ray flashes observed up to 20 MeV, Science, 307 (5712), 1085-1088.

Stanley, M. A., X.-M. Shao, D. M. Smith, L. I. Lopez, M. B. Pongratz, J. D. Harlin, M. Stock, and A. Regan (2006), A link between terrestrial gamma-ray flashes and intracloud lightning discharges, Geophys. Res. Lett., 33, L06803, doi:10.1029/2005GL025537.

Stenbaek-Nielsen, H. C., T. Kanmae, M. G. McHarg, and R. Haaland (2013), High-speed observations of sprite streamers, Surv. Geophys., 34, 769-795, doi:10.1007/s10712-013-9224-4.

Stolzenburg, M., T. C. Marshall, S. Karunarathne, N. Karunarathna, L. E. Vickers, T. A. Warner, R. E. Orville, and H.-D. Betz (2013), Luminosity of initial breakdown in lightning, J. Geophys. Res. Atmos., 118, 2918-2937, doi:10.1002/jgrd.50276.

Tavani, M., et al. (2011), Terrestrial gamma-ray flashes as powerful particle accelerators, Phys. Rev. Lett., 106(1), 018501, doi:10.1103/PhysRevLett.106.018501.

Uman, M. A. (2001), The Lightning Discharge, Dover, Mineola, N. Y.

Vallance Jones, A. V. (1974), Aurora, D. Reidel, Norwell, Mass.

Van Zyl, B., and W. Pendleton Jr. (1995), $\mathrm{N}_{2}^{+}(X), \mathrm{N}_{2}^{+}(A)$, and $\mathrm{N}_{2}^{+}(B)$ production in $\mathrm{e}^{-}+\mathrm{N}_{2}$ collisions, J. Geophys. Res., 100(A12), $23,755-23,762$

Winn, W. P., G. D. Aulich, S. J. Hunyady, K. B. Eack, H. E. Edens, P. R. Krehbiel, W. Rison, and R. G. Sonnenfeld (2011), Lightning leader stepping, $K$ changes, and other observations near an intracloud flash, J. Geophys. Res., 116, D23115, doi:10.1029/2011JD015998.

Xu, W., S. Celestin, and V. P. Pasko (2012), Source altitudes of terrestrial gamma-ray flashes produced by lightning leaders, Geophys. Res. Lett., 39, L08801, doi:10.1029/2012GL051351. 